\title{
The determination of vasoactive substances during autonomic dysreflexia
}

\author{
GC Zhou ${ }^{1}, \mathrm{~J} \mathrm{Yu}^{2}, \mathrm{HH}$ Tang ${ }^{1}$ and $\mathrm{J} \mathrm{Shi}^{1}$ \\ ${ }^{1}$ Department of Spine and Spinal Cord Surgery; ${ }^{2}$ The Institute of Basic Science for Rehabilitation Medicine, The \\ China Rehabilitation Research Centre, Beijing 100077, China
}

\begin{abstract}
Urinary bladder percussion induced autonomic dysreflexia (AD) was observed in spinal cord injured patients with a complete neurological lesion, the upper level being above T5. To document the pathology and study the etiology of autonomic dysreflexia to further investigate its mechanism, this paper presents some clinical data on the determination of vasoactive substances such as norepinephrine (NE), epinephrine (E), renin (R), angiotensin II (AII) and atrial natriuretic polypeptide (ANP) before and during bladder percussion in 30 patients with a thoracolumbar or cervical spine and spinal cord injury. It is demonstrated that tapping the urinary bladder of such patients can cause AD. Changes of some of the vasoactive substances in the plasma were also observed, which might indicate that autonomic dysreflexia result from excitation of the sympathetic nervous system. Overactivity of the sympathetic nervous system was antagonized by excitation of the vegetative nerve system. There was no correlation between changes of blood pressure and adrenal function as well as the change of R-A II system; during autonomic dysreflexia, the inclement of ANP secretion played an important role in recovering homeostasis.
\end{abstract}

Keywords: autonomic dysreflexia; vasoactive substances; paraplegia; tetraplegia; spinal cord injury

\section{Introduction}

Autonomic dysreflexia is an acute syndrome of massive unchecked reflex sympathetic discharge that can occur in patients with a spinal cord lesion above the major sympathetic splanchnic outflow. ${ }^{1}$

Cardiovascular function becomes abnormal and unstable after such a spinal cord injury (SCI), especially in patients with the lesion of the cord above T5. This is often characterised by episodes of hypertension, severe headache, bradycardia secondary to baroreceptor reflexes and sweating. These exaggerated reactions are generally considered to be an overaction of the sympathetic nerves below the lesion of spinal cord which could not be regulated by the descending inhibitory control from the vasomotor center in the brain stem. ${ }^{2,3}$ To elucidate the mechanism of autonomic dysreflexia some investigations have been performed during autonomic dysreflexia to test NE which was formed and released by the sympathetic nerve endings. It is shown that the level of $\mathrm{NE}$ in the plasma increased during AD, which indicated that $\mathrm{AD}$ is closely related to the overactivation of the sympathetic nerves below the lesion in the spinal cord. ${ }^{4}$ Krum tested vasoactive substances during bladder distension following slow bladder filling and found that there were no significant changes of NE, R, aldosterone, vasopressin and ANP in plasma during bladder distension to account

Correspondence: GC Zhou for the blood pressure rise in patients with a high spinal cord lesion. 5 The purpose of this study is to investigate the contribution of vasoactive substances in relation to autonomic dysreflexia to enable an understanding of its pathophysiology.

\section{Materials and methods}

\section{Population}

Thirty patients with complete spinal cord injury were studied. They were divided into two groups: 15 patients with paraplegia due to a thoracolumbar SCI and 15 patients with tetraplegia resulting from a cervical SCI.

The physical condition of all of the patients in our study showed that they were in a stable state and none had hypertension, pressure sores, ectopic ossification or deep venous thrombosis nor did they have a urinary tract infection or a contracted bladder and renal function was normal. Reflex micturition in these patients had formed. For controls, ten healthy young volunteers participated in the study (Table 1).

\section{Method}

All of the patients in the study were catheterised with a Folatex balloon catheter with an external diameter of $3.3 \mathrm{~mm}$ and were in a supine position for $2 \mathrm{~h}$. The blood pressure (BP) and pulse rate (PR) were measured 
in the right upper arm by a Dynamic 500 vital sign monitor. The empty bladder of each patient was then slowly infused with $250 \mathrm{ml}$ of normal saline, kept at room temperature, through the catheter with an infusion rate of $25 \mathrm{ml} / \mathrm{min}$. Blood samples were then taken from the right femoral vein for the measurement of NE, E, R, AII and ANP.

To determine NE and E, blood samples were collected into chilled heparin tubes. Plasma was immediately separated by centrifugation at $4{ }^{\circ} \mathrm{C}$ and stored at $-80^{\circ} \mathrm{C}$ and tested within 5 days. The concentration of $\mathrm{NE}$ and $\mathrm{E}$ in the plasma was determined by high performance liquid chromatography (HPLC) using Model 460 electrochemical detector (Waters, Eschborn, Germany) and N-510 pump. Biophase ODS $5 \mu \mathrm{M}, 4.6 \times 250 \mathrm{~mm}$ column was used. The mobile phase consisted of $0.05 \mathrm{M}$ acetate buffer (pH 3.5), $0.6 \mathrm{mM}$ SOS reagent, $1 \mathrm{mM}$ EDTA and 1\% methanol. Concentrations of detected compounds were PC calculated which were related to external standards and multiplied with the recovery factor of an internal standard. For the determination of R and AII, blood samples were collected into tubes containing an enzyme inhibitor (composed of $0.3 \mathrm{M}$ EDTA-2Na, $0.34 \mathrm{M}$ 8-mercaptoquinoline and $0.32 \mathrm{M}$ dimercaprol) and kept at $4{ }^{\circ} \mathrm{C}$. The plasma was separated by centrifugation and stored at $-80^{\circ} \mathrm{C}$ and tested within 2 months. The concentration of R, AII and ANP in plasma was assayed using radioimmunoassay kits provided by the Northern Institute of Immunological Reagents, China Isotope Corporation and measured in a LKB-1275 Minigama counter.

Ten volunteers in the control group also rested in bed for $2 \mathrm{~h}$ until the bladder could be palpated in the suprapubic area. BP and PR were measured and recorded in the same way and blood samples were also studied.

Bladder percussion was performed at the suprapubic area with index and middle fingers for at least 3 min continuously; and the $\mathrm{BP}$ and PR were

Table 1 Contribution of tested objects

\begin{tabular}{lccccc}
\hline & Age & \multicolumn{2}{c}{ Sex } & \multicolumn{2}{c}{ Course } \\
Patients & $(\times \pm \mathrm{SD})$ & Female & Male & $<6 M$ & $>6 M$ \\
\hline Paraplegic & $32.2 \pm 11$ & 2 & 13 & 12 & 3 \\
Tetraplegic & $25.2 \pm 9.9$ & 3 & 12 & 14 & 1 \\
Healthy & 19 to 31 & & 10 & & \\
\hline
\end{tabular}

measured and recorded every $30 \mathrm{~s}$. If there was no change in the BP and PR the percussion was stopped and a blood sample was taken for study. Otherwise, bladder percussion was halted until the BP and PR values were no longer raised and reached a plateau (ie the recorded value remained constant for two continuous readings). The highest $\mathrm{BP}$ and the lowest PR were recorded and the blood sample was taken at the highest BP. The occurrence of AD was considered when the PR reduced ten times per minute, the systolic and diastolic blood pressures (SBP and DBP) rose to $4 \mathrm{Kpa}$ and $3 \mathrm{Kpa}$ respectively. The ten heathly young men controls were studied in the same way.

The statistical analysis of clinical data was processed by the Student $t$ test using SDAS Ver. 2.0 for PC.

\section{Results}

Measure of blood pressure and pulse rate

None of the patients in the study had any change of BP and PR before bladder percussion although they underwent catheterisation and infusion with $250 \mathrm{ml}$ of normal saline. During bladder percussion, the BP and PR of the control group and that of the paraplegic patients remained quite stable. Although the SBP of the paraplegic group was higher than that of other groups, there were no significant differences. Tetraplegic patients, however, appeared to have the typical AD syndrome with an increase of BP and a decrease of PR. Some had headache, sweating and goose skin. A comparison of SBP, DBP and PR before and during bladder percussion in the three groups is demonstrated in Table 2.

\section{Determination of vasoactive substances}

Before bladder percussion, the concentrations of vasoactive substances in the patients and normal volunteers were similar $(P>0.05)$. During bladder percussion, the NE concentration in the plasma of SCI patients showed a clear increase compared with that of the control group $(P<0.05)$. The NE value of tetraplegic patients showed even more augmentation compared with the NE value of the paraplegic patients $(P<0.05)$. Only tetraplegic patients showed an obvious increase of $\mathrm{R}$-value $(P<0.05)$ (Table 3$)$.

Table 2 Measurements of BP (KPa), and PR (beats/min)

\begin{tabular}{|c|c|c|c|c|c|c|}
\hline \multirow[b]{2}{*}{ Patients } & \multicolumn{3}{|c|}{ Before percussion } & \multicolumn{3}{|c|}{ During percussion } \\
\hline & $S B P$ & $D B P$ & $P R$ & $S B P$ & $D B P$ & $P R$ \\
\hline Tetraplegic & $15.6 \pm 1.6$ & $8.9 \pm 1.3$ & $74.8 \pm 20.6$ & $22.4 \pm 4.0 *$ & $13.0 \pm 2.7 *$ & $61.0 \pm 16.3^{*}$ \\
\hline Paraplegic & $16.6 \pm 1.4$ & $9.2 \pm 1.3$ & $79.1 \pm 12.9$ & $16.1 \pm 1.1$ & $9.3 \pm 2.4$ & $81.8 \pm 13.3$ \\
\hline Healthy & $15.6 \pm 1.7$ & $9.6 \pm 1.4$ & $72.6 \pm 14.3$ & $16.0 \pm 1.4$ & $9.8 \pm 2.1$ & $75.2 \pm 10.4$ \\
\hline
\end{tabular}

$* P<0.05$ 
Table 3 Concentrations of vasoactive substances in blood samples (pg/ml)

\begin{tabular}{cccccc}
\hline Measured & Patients & $N E$ & $E$ & $R$ & $A N P$ \\
\hline Before the & Tetraplegic & $164.8 \pm 85.8$ & $123.7 \pm 35.0$ & $64.0 \pm 65.0$ & $31.2 \pm 16.6$ \\
bladder & Paraplegic & $165.9 \pm 97.2$ & $122.6 \pm 46.0$ & $55.0 \pm 22.0$ & $36.4 \pm 16.6$ \\
percussion & Healthy & $164.0 \pm 84.4$ & $121.0 \pm 16.0$ & $56.0 \pm 44.0$ & $35.7 \pm 17.0$ \\
After the & Tetraplegic & $239.1 \pm 119.0^{*}$ & $138.8 \pm 51.3$ & $115.0 \pm 81.0 *$ & $34.5 \pm 18.4$ \\
bladder & Paraplegic & $219.0 \pm 100.0^{*}$ & $133.7 \pm 35.0$ & $59.0 \pm 43.0$ & $42.6 \pm 13.0$ \\
percussion & Healthy & $180.0 \pm 98.4$ & $132.9 \pm 30.0$ & $58.0 \pm 45.0$ & $41.4 \pm 24.0$ \\
\hline
\end{tabular}

$* P<0.05$

\section{Discussion}

The results of the study showed that urinary bladder percussion could cause a rise in BP and a decrease in PR in patients with a cervical spinal cord injury. As had been previously demonstrated by Wurster ${ }^{6}$ and Mathias ${ }^{7}$ the increase of blood pressure resulted from the exaggerated activation of the sympathetic nerves below the lesion of the spinal cord. Sternberg et al recorded muscle nerve sympathetic activity using a very sensitive technique with a direct microneuroelectrode. ${ }^{2}$ Mathias et al reported that stimulation of the bladder in high spinal cord injured patients could result in AD with a simultaneous increase of $\mathrm{NE}$ concentration in plasma. ${ }^{4}$

In the present study, bladder percussion caused a significant increase of NE concentration in the plasma of both paraplegic and tetraplegic groups $(P<0.05)$. The NE concentration in the plasma of the cervical spinal cord injured patients was even much higher than was found in the paraplegic patients $(P<0.05)$. This result indicated that when bladder percussion was performed on SCI patients an overaction of the sympathetic nerves below the cord lesion could occur and tetraplegic patients showed an even more intense and extensive response of the sympathetic nervous system; and this overactivation of the sympathetic nerves resulted in AD. These results are in good correspondence with those of Mathias but differ from Krum's results. ${ }^{4,5}$

NE formed in the synaptic vesicles of sympathetic terminals was a conduction mediator of neural transmission of the sympathetic nerve system. Formed NE closely bound to ATP chromogranin and stored in vesicles of terminals. With the impulse, the calcium channel of the presynaptic membrane opened, calcium cations entered cells impelling the movement of vesicles towards the presynaptic membrane causing fusion of the two. NE was released and entered into the synaptic cleft and then bound with the receptors on the postsynaptic membranes before the performance of its biological function. NE in the synaptic cleft could be reabsorbed by the terminals into vesicles and restored again. It was also taken up by vascular smooth muscle cells, cardiac muscle cells and vascular endotheliocyte cells and part of it would be deactivated in the synaptic cleft by catechol-O-methyltransferase
(COMT). The remainder entered the circulation which was clinically taken as a measure of the active state of the sympathetic nervous system.

The NE entered the blood from the synaptic cleft with no cardiovascular effect. It was immediately deactivated by COMT. Mathias and his coworkers found that after an intravenous injection of $\mathrm{NE}$ into the cervical spinal cord injured patient an AD type of increase of the blood pressure would not occur unless the NE concentration in bloods reached 21 fold of the normal value. ${ }^{4}$ In our study with tetraplegic patients, the NE concentration was $164.8 \pm 85.5 \mathrm{pg} / \mathrm{ml}$ in resting condition and was only 1.5 times when AD occurred. According to Mathias' study, this concentration of NE in the plasma was still not enough to cause an increase in BP. The result of our study indicated that AD was not only induced by the overreaction of the sympathetic nerves below the lesion of the cord. As some authors considered that in those who were tetraplegic, vessels caudal to the injury level were more sensitive to $\mathrm{NE}$ and therefore there was even more prominent AD features.

In our study we found that BP of paraplegic patients had no obvious changes although there was autonomic overactivity below the injury level and an increase of NE concentration in blood. As Guttmann and Whitteridge reported ${ }^{8} \mathrm{AD}$ related to the injury level of the spinal cord. The higher the injury level the more obvious was the change of BP during AD and vice versa. With a lesion caudal to T10 there was no consistent BP elevation for the resolution of supraspinal reflex vasomotion. ${ }^{8}$

During bladder percussion, the renin concentration in the plasma arose significantly in the tetraplegic group. This result differed from some previous reports. ${ }^{5,9,10}$ In those investigations, the concentration of renin fell during bladder stimulation. It was considered that the reduction of the renin level in the plasma after bladder stimulation might be a reflex fall in response to the elevated blood pressure. $5,9,10$ Renin is closely associated with angiotensin which is called renin-angiotensin-system (RAS). It was responsible for the regulation of vasomotor activity, renin was formed in the juxtaglomerular apparatus. It was secreted more quickly when the sodium in the blood and the circulation volume decreased or when the sympathetic nerves (greater and lesser splanchic nerves 
composed by T5-T10) of the juxtaglomerular apparatus were excited. When bladder percussion was performed on the tetraplegic patients, stimulation of that nervous system occurred which caused an increase of $\mathrm{R}$ secretion and then an increase of $\mathrm{R}$ concentration in the plasma. Bladder percussion did not cause excitation of the greater and lesser splanchnic nerves in normal controls or in paraplegic patients with a lower spinal cord injury level caudal to T5. The only biological function of $R$ was to transform angiotensin $\mathrm{I}(\mathrm{AI})$ and the latter was then enzymatically converted to angiotensin II (AII) in the lungs, liver and kidneys. AII was a powerful vasoactive substance and it was $8-40$ times more effective than $\mathrm{NE}$ on raising the blood pressure.

Epinephrine was only formed and released in the adrenal medulla. The bladder percussion on paraplegic patients did not cause a change of $\mathrm{E}$ concentration in the plasma. This result, was similar to Mathias ${ }^{4}$ and indicated that there was no overactivation of adrenal glands during AD.

During AD the splanchnic vessels were markedly constricted and a large volume of blood poured into the circulation causing an increase of BP. Therefore, a neurological regulatory mechanism was considered in maintaining the homeostasis: the stimulation of the baroreceptor of aortic arch and carotid sinus followed by stimulation of the vagus nerves and decrease of the pulse rate. The increase of atrial natriuretic polypeptide secretion was also a very important factor in the body fluid regulation mechanism. ANP was essentially derived from myocardial cells, especially from the myocardium of the right atrium. ${ }^{11,13}$ The increase of the circulation volume and blood pressure induced an increased secretion of ANP, which played an important role during AD in regulating and maintaining the homeostasis by the following ways: (1) diuresis: by increasing the filtration rate of glomerulus and inhibiting the reabsorption rate of the renal tubules which led to a decrease of the circulation volume; (2) acting as an antagonist to some vasoactive substances such as NE, by dilating vessels to reduce the resistance of vessels, which led to a decrease of the blood pressure; (3) reducing the cardiac output and the index of cardiac ejection; (4) inhibiting the delivery of NE from the terminals of sympathetic nerve by blocking calcium channels. ${ }^{14-19}$

In summary, our study demonstrated that there was a major difference in the alteration of $\mathrm{BP}$ and PR caused by bladder percussion between patients with tetraplegia and those with paraplegia. Changes of vasoactive substances during bladder percussion indicated that: (1) AD resulted from overaction of the sympathetic nervous system below the cord lesion and the increase of vascular sensitivity to NE; (2) the adrenal glands and the renin-angiotensin system did not respond to the rise of BP; (3) ANP might play a very important role in maintaining homeostasis during AD besides the parasympathetic nervous system.

\section{References}

1 Kurnick NB. Autonomic hyperreflexia and its control in patients with spinal cord lesions. Ann Intern Med 1956; 44: 678-686.

2 Sternberg L, Blumberg H, William BG. Sympathetic activity in man after spinal cord injury. Outflow to muscle below lesion. Brain 1986; 109: 695-715.

3 Rolland P, Erikson MD. Autonomic hyperreflexia: pathophysiology and medical management. Arch Phys Rehabil 1980; 61: $430-440$.

4 Mathias CJ et al. Plasma catecholamines during proximal neurogenic hypertension in quadriplegia. Circulation Res 1976; 39: $204-208$

5 Krum $\mathrm{H}$ et al. Cardiovascular and vasoactive hormone responses to bladder distension in spinal and normal man. Paraplegia 1992; 30: $348-354$

6 Wurster RD, Randall WC. Cardiovascular responses to bladder distension in patients with spinal transaction. Am J Physiol 1975; 228: $1288-1292$.

7 Mathias CJ, Frankel HL, Christensen NJ, Spalding JMK. Enhanced pressor responses to noradrenaline in patients with cervical spinal cord transaction. Brain 1986; 99: 757-770.

8 Guttmann L, Whitteridge D. Effects of bladder distension on autonomic mechanism after spinal cord injury. Brain 1974; 70: $361-404$

9 Nanninga JB, Rosen JS, Krumlovsky F. Effect of autonomic hyperreflexia on plasma renin. Urol 1976; 7: 638-640.

10 Mathias CJ et al. Renin and aldosterone release during sympathetic stimulation in tetraplegia. Clin Sci 1981; 60: $399-$ 404.

11 Tang $\mathbf{J}$ et al. Charaterization and localization of atriopeptin in rat atrium. Peptides 1984; 5: 1173.

12 Edwards BS et al. Atrial stretch, not pressure, is the principal determinant controlling the acute release of ANP. Cir Res 1988; 62: $191-195$.

13 Kinnunen P, Vuolteenaho O, Ruskoaho H. Mechanisms of atrial and brain natriuretic peptide release from rat ventricular myocardium: effect of stretching. Endocrinol 1993; 132: $1961-$ 1969.

14 Laragh JH, Atlas SA. Atrial natriuretic hormone: a regulator of blood pressure and volume homeostasis. Kidney Int 1988; 34 Suppl. 25: $64-71$.

15 Richards AM et al. Atrial natriuretic hormone has biological effects in man at physiological plasma concentrations. J Clin Endocrinol Metab 1988; 67: 1134-1139.

16 Granger JP, Opgenorth TJ, Salazar J. Long term hypertensive and renal effects of atrial natriuretic peptide. Hypertension 1968 ; 8 Suppl. II: $112-116$.

17 Debinski W et al. Atrial natriuretic factor partially inhibits the stimulated catecholamine synthesis in superior cervical ganglia of the rat. Neurosci Lett 1987; 77: $92-96$.

18 Tsuda $\mathrm{K}$ et al. Effects of alpha-human atrial natriuretic peptide on neurosecretion and vascular responsiveness in spontaneously hypertensive rats. J Hypertens 1986; 4: S331-S333.

19 Anderson JV et al. Atrial natriuretic peptide: Evidence of action as a natriuretic hormone at physiological plasma concentrations in man. Clin Sci 1987; 72: 305-312. 